\title{
ALOE VERA ETHANOL EXTRACT AS A THERAPY FOR ALVEOLAR MANDIBLE REGENERATION IN LIPOPOLYSACCHARIDE-EXPOSED RATS
}

\author{
LATIFAH FITRIANI RAHMAN ${ }^{1}$, OCTAVIANA WIDYA PANGESTIKA ${ }^{1}$, DELVI FITRIANI ${ }^{1 *}$, \\ RUDHANTON RUDHANTON ${ }^{2}$, NUR PERMATASARI ${ }^{3}$
}

${ }^{1}$ Department of Academic Faculty of Dentistry, University of Brawijaya, Malang 65145, Indonesia. ${ }^{2}$ Department of Periodontia, Faculty of Dentistry, University of Brawijaya, Malang 65145, Indonesia. ${ }^{3}$ Department of Oral Biology, Faculty of Dentistry, University of Brawijaya, Malang 65145, Indonesia. Email: delfifitriani@yahoo.com

Received: 29 June 2018, Revised and Accepted: 08 August 2018 and 15 November 2018

ABSTRACT

Objective: The study examined the effects of Aloe vera ethanol extract on alveolar mandible regeneration in rats (Rattus norvegicus).

Methods: Rats were divided into five groups as follows: Negative control group received no treatment, positive control group received Escherichia coli lipopolysaccharide (LPS) alone for 5 days, and Groups P1, P2, and P3 received LPS for 5 days followed by 40, 80, and $160 \mathrm{mg} / \mathrm{kg}$ BW A. vera ethanol extract, respectively, orally on days 6-12. Alveolar bone tissues were taken and histologically processed through hematoxylin-eosin staining. Osteoblast and osteoclast numbers in alveolar tissue were also examined.

Results: One-way ANOVA revealed strong relationships between the $A$. vera ethanol extract dosage and the numbers of osteoblasts $(\mathrm{r}=0.921, \mathrm{p}<0.05)$ and osteoclasts $(\mathrm{r}=-0.631, \mathrm{p}<0.05)$ in rats.

Conclusion: A. vera ethanol extract appears capable of stimulating alveolar bone regeneration following LPS exposure.

Keywords: Aloe chinensis, Baker ethanol extract, Lipopolysaccharide, Osteoblasts, Osteoclasts, Alveolar bone.

(c) 2018 The Authors. Published by Innovare Academic Sciences Pvt Ltd. This is an open access article under the CC BY license (http://creativecommons. org/licenses/by/4. 0/) DOI: http://dx.doi.org/10.22159/ijap.2018.v10s1.03

\section{INTRODUCTION}

Periodontal disease is common and widespread and can affect children, adults, and the elderly. The 2003 World Oral Health Report identified periodontal disease as the fourth most expensive disease to treat. The Scheffler survey revealed that $75 \%$ of the American population has periodontal disease. The prevalence of periodontal disease in all age groups in Indonesia has reached $96.58 \%$ [1], whereas data from the Department of Health of Malang indicated that periodontal disease is the seventh most common disease in the state [2].

Periodontitis, a cause of tooth loss in adults, is a chronic infection of tooth-supporting tissues including the gingiva, periodontal ligament, bone, and cementum by bacteria. Substances produced by bacteria cause tissue inflammation and progressive alveolar bone damage, which are the main characteristics of periodontitis [3]. The major microbes that cause periodontitis are Porphyromonas gingivalis, Prevotella intermedia, and Actinobacillus actinomycetemcomitans; however, other microbes including Gram-negative bacteria such as Escherichia coli, Fusobacterium nucleatum, and Actinomyces israelii can also trigger periodontitis [4].

Gram-negative bacterial species produce lipopolysaccharide (LPS), a structural component of the outer membrane that protects bacteria against host immune defenses [5]. LPS induces the production of local factors, namely pro-inflammatory cytokines such as interleukin$1 \alpha$ (IL- $1 \alpha)$, IL- $1 \beta$, tumor necrosis factor- $\alpha$ (TNF- $\alpha$ ), and eicosanoids, including prostaglandin E2 (PGE2). Prostaglandins and proinflammatory cytokines promote destruction of periodontal tissue by stimulating osteoclast production and activity and decreasing the numbers and activity of osteoblasts [6]. Umezu et al. revealed that mice injected with $E$. coli LPS in the mucosal region of the maxillary first molar exhibit alveolar bone resorption caused by excessive osteoclast numbers and activity.
Alveolar bone regeneration is a suitable treatment goal for patients with periodontitis and severe bone destruction. Regenerative periodontal tissue healing occurs through the formation of new periodontal tissues, particularly the alveolar bone, functional periodontal ligament, and cementum. Increased osteoclastogenesis is one indicator of bone regeneration [7].

Several studies previously reported the efficacy of Aloe vera (Aloe chinensis) in healing bone defects. A. vera is considered to express biogenic stimulators and wound healing hormones because it promotes cell regeneration. A. vera contains acetylated mannose (acemannan), a large polysaccharide that promotes the formation of collagen type 1 fibers and acts as an immunostimulator enhancing T-helper immune responses against intracellular pathogens such as viruses, bacteria, and parasites. Another study conducted by Jittapiromsak et al. postulated that acemannan can stimulate bone morphogenetic protein-2 (BMP2) expression in pulpal fibroblasts and periodontal tissues, thus resulting in bone regeneration. Furthermore, Kresnohadi combined A. vera and xenograft cancellous grafts, thus observing increases in BMP2 expression and osteoblast counts as well as decreased receptor activator of nuclear factor kappa-B ligand (RANKL) expression, which is an indicator of decreased osteoclast production [8].

Most current studies have found that molecular signals trigger the formation of complex tissues. Molecular biology studies identified BMP as a bone differentiation initiator. BMP regulates cartilage and bone differentiation as well as bone growth through progenitors that trigger osteoblast formation [9].

Thus, the effects of A. chinensis baker ethanol extract on LPSinduced alveolar bone destruction were examined in male rats (Rattus norvegicus) based on osteoblast and osteoclast counts. 


\section{METHODS}

\section{Research design}

This in vivo study was randomized and controlled in nature.

\section{Samples}

The study samples included male rats maintained in the Pharmacology Laboratory of the Faculty of Medicine, Brawijaya University, Malang. The rats were 2 months old, healthy, active with normal behavior and weighed 200-250 g. Rats used in previous studies, those with a lack of appetite, those in poor condition as well as dead rats were excluded from the study.

\section{Variables}

The study variables were as follows:

a. Independent variable: $A$. vera ethanol extract;

b. Dependent variable: Number of osteoclasts;

c. Control: Simple criteria, method for administering LPS, and method for administering $A$. vera extract.

Six repetitions were performed. The rats were divided into five groups: A negative control group that received no treatment, a positive control group that received LPS alone, and three experimental groups treated with LPS followed by 200,400 , or $800 \mathrm{mg} / 200 \mathrm{~kg} \mathrm{BW}$ of $A$. vera ethanol extract.

\section{Setting and time of the study}

The study was conducted at the Pharmacology and Anatomical Pathology Laboratory of the Faculty of Medicine, Brawijaya University, between December 2014 and February 2015.

\section{PROCEDURE}

\section{Ethical clearance}

The study was ethically approved by the Medical Research Ethics Commission of the Faculty of Medicine, Brawijaya University.

\section{Sample preparation}

Male rats were weighed using an analytical scale. The rats were allowed to adapt to the environment for 1 week and maintained in $40 \mathrm{~cm} \times 30 \mathrm{~cm} \times 30 \mathrm{~cm}^{3}$ cages. Each cage included no more than two animals.

\section{Dosage conversion}

a. LPS dosage $=5 \mu \mathrm{g} / 0.05 \mathrm{ml}$ of PBS,

b. A. vera.

The conversion factor between a $70 \mathrm{~kg}$ man and $200 \mathrm{~g}$ mouse was 0.018 . The recommended A. vera dosage for humans is $10-15 \mathrm{~g} /$ day. Thus, the A. vera dose for rats was calculated as follows:

$=0.018 \times 10 \mathrm{~g}$

$=0.18 \mathrm{~g} / 200 \mathrm{~g} \mathrm{BW}$

$=180 \mathrm{mg} / 200 \mathrm{~g} \mathrm{BW} \approx 36 \mathrm{mg} / \mathrm{kg} \mathrm{BW}$.

The extract was prepared at three different dosages:

- $40 \mathrm{mg} / \mathrm{kg}$ BW for Group A

- $80 \mathrm{mg} / \mathrm{kg}$ BW for Group B

- $160 \mathrm{mg} / \mathrm{kg}$ BW for Group C.

\section{Sample grouping}

a. Group 1: Negative control group (K-) that was not administered LPS or $A$. vera ethanol extract:

b. Group 2: Positive control group (K+) administered LPS alone;

c. Group 3: (P1) Group administered LPS followed by $40 \mathrm{mg} / \mathrm{kg} \mathrm{BW}$ A. vera ethanol extract;

d. Group 4: (P2) Group administered LPS followed by $80 \mathrm{mg} / \mathrm{kg} \mathrm{BW}$ A. vera ethanol extract;

e. Group 5: (P3) Group administered LPS followed by $160 \mathrm{mg} / \mathrm{kg} \mathrm{BW}$ A. vera ethanol extract.

Material preparation

Material used during the experiment included E. coli LPS (Sigma) to induce periodontitis and $A$. vera ethanol extract to stimulate alveolar bone repair.

\section{LPS preparation}

Totally, $10 \mathrm{mg}$ of LPS was dissolved in $2 \mathrm{ml}$ of PBS and stored in a sealed container at room temperature.

\section{A. vera ethanol extract preparation}

The A. vera ethanol extract was obtained through maceration. The process required approximately $200-400 \mathrm{~g}$ of $A$. vera powder as the main raw material, $200 \mathrm{ml}$ of $96 \%$ ethanol for polysaccharide deposition, a deposition time of $10 \mathrm{~h}$, and a precipitation temperature of $10^{\circ} \mathrm{C}$. The preparations were then filtered into a porcelain bowl and allowed to stand uncovered at room temperature for 1 day. Following evaporation, the extract was stored in a container in a cool place and protected from sunlight [10].

\section{EXPERIMENTS}

\section{Sedating the animals}

Before treatment, the rats were sedated by administering an injection of ketamine (KTM 100; $40 \mathrm{mg} / \mathrm{kg} \mathrm{BW}$ ) into the right hind leg.

\section{Applying the materials}

LPS $(5 \mu \mathrm{g} / 0.05 \mathrm{ml})$ was injected into the first incisive tooth gingival sulcus located on the mandibular right labia. PBS $(0.02 \mathrm{ml})$ was injected using a 30-G insulin needle once daily for 5 days. The first incisor was selected because it is categorized as a front tooth and is, therefore, more visible than the other teeth.

Using a feeding tube, $A$. vera ethanol extract ( $1 \mathrm{ml}$ ) was administered for 7 days starting on day 6 .

\section{Alveolar mandible surgery}

The surgery was conducted after 7 days of treatment with $A$. vera.

The rats were sedated using KTM $(80 \mathrm{mg} / \mathrm{kg}$ BW), and their alveolar mandible bone was horizontally cut. The dead animals were then cleaned, sterilized using $70 \%$ alcohol, and buried into a $100 \mathrm{~cm} \times 30 \mathrm{~cm} \times 50 \mathrm{~cm}^{3}$ hole in the backyard of the Pharmacology Laboratory. Each hole contained 24 dead rats. The mandibles were preserved using $10 \%$ formalin for $24 \mathrm{~h}$ and $10 \%$ EDTA for 14 days to decalcify the tissue. The solution was changed every $24 \mathrm{~h}$, and the bones were washed under running water.

\section{Paraffin block preparation}

Tissues were dehydrated using acetone for $24 \mathrm{~h}$ and cleared using xylol twice for $1 \mathrm{~h}$ each. Next, infiltration was done using soft paraffin at $42^{\circ} \mathrm{C}-46^{\circ} \mathrm{C}$ twice for $1 \mathrm{~h}$ each followed by blocking with hard paraffin at $46^{\circ} \mathrm{C}-52^{\circ} \mathrm{C}$ for $1 \mathrm{~h}$. Afterward, the tissue was cut vertically using a $4-6-\mu \mathrm{m}$ microtome rotary and then heated at $60^{\circ} \mathrm{C}$. The tissue was then soaked in xylol twice for 5 min each followed by a series of different concentrations of alcohol (95\%, 85\%, 70\%, 50\%, and 30\%) twice for 3 min each.

For hematoxylin-eosin staining, tissues were incubated in Harris hematoxylin for $15 \mathrm{~min}$ followed by acid alcohol drops for 3-10 s and ammonium solution for 3-10 s. This was followed by counterstaining for 15-20 s and dehydrating using the ethanol series. Then, tissues were exposed to xylol for $5 \mathrm{~min}$ and mounted for observation using a digital microscope (five fields, $\times 400$ ) to count the numbers of osteoblasts and osteoclasts with the assistance of an anatomical pathology lecturer and laboratory analyst.

\section{Data analysis}

Normality was assessed using the Shapiro-Wilk test because the number of samples exceeded 50. A two-tailed t-test was used to compare scores between two groups, with $\mathrm{p}<0.05$ indicating statistically significant differences. Levene's test was used to compare normality and variance homogeneity between the positive control and experimental groups. When the data were normally distributed $(p>0.05)$ and variance homogeneity was accepted $(p>0.05)$, one-way ANOVA was conducted for hypothesis testing. Post hoc testing was then 
used to identify significant differences based on ANOVA. Finally, the correlation-regression test was performed to assess the relationship between the A. vera dosage and osteoblast and osteoclast counts.

\section{RESULTS}

Figs. 1 and 2 show that osteoblast numbers were the lowest in the negative control group, whereas osteoclast counts were the highest in this group. Meanwhile, osteoblast counts increased with an increasing dosage of the A. vera ethanol extract; however, the opposite was seen in the case of osteoclasts. Figs. 3 and 4 show histological comparison of osteoblasts and osteoclast counts.

The Shapiro-Wilk test demonstrated that the data for osteoblasts and osteoclasts were normally distributed $(\mathrm{p}<0.05)$. The Levene's homogeneity test illustrated that the variance was homogenous in both osteoblast $(\mathrm{p}=0.336)$ and osteoclast data $(\mathrm{p}=0.700)$. Meanwhile, oneway ANOVA revealed significant differences in osteoblast $(p=0.000)$ and osteoclast counts ( $\mathrm{p}=0.012$ ) based on the $A$. vera ethanol extract dosage.

The results of post hoc testing using the least significant difference test revealed significant differences between the control and experimental groups as well as among the experimental groups regarding the numbers of osteoblasts and osteoclasts.

The Pearson correlation test was employed to assess the correlation between the $A$. vera extract dosage and the numbers of osteoblasts

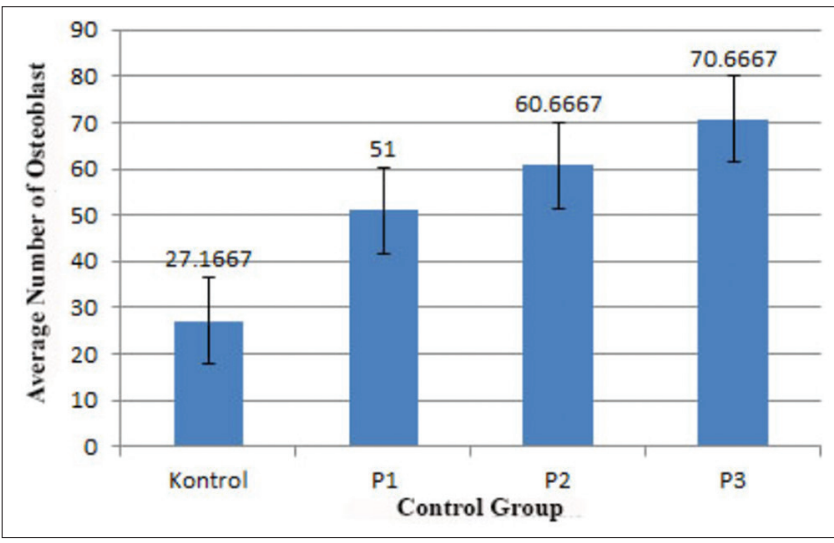

Fig. 1: Average number of osteoblasts in the groups. Description: K/P0: Lipopolysaccharide (LPS) treatment alone, P1: LPS treatment followed by $40 \mathrm{mg}$ of Aloe chinensis baker ethanol extract, P2: LPS treatment followed by $80 \mathrm{mg}$ of A.chinensis baker ethanol extract, P3: LPS treatment followed by $160 \mathrm{mg}$ of $A$. chinensis baker ethanol extract

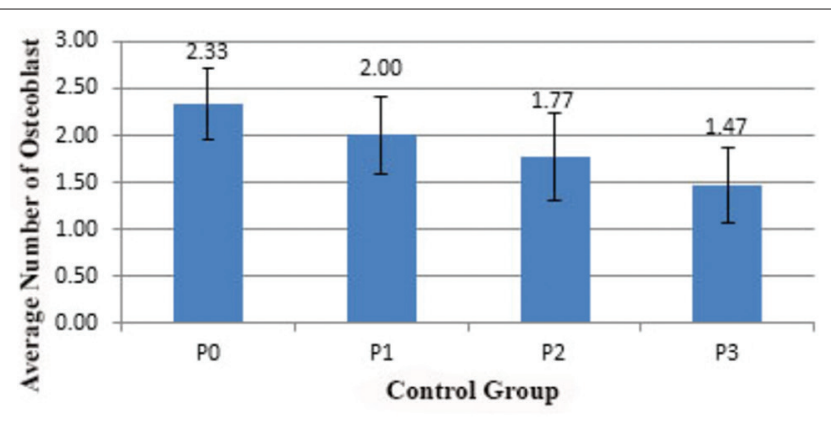

Fig. 2: Average number of osteoclasts in the groups.

Description: K/P0: Lipopolysaccharide (LPS) treatment alone. P1: LPS treatment followed by $40 \mathrm{mg}$ of Aloe chinensis baker ethanol extract, P2 LPS treatment followed by $80 \mathrm{mg}$ of A. chinensis baker ethanol extract, P3 LPS treatment followed by $160 \mathrm{mg}$ of $A$. chinensis baker ethanol extract and osteoclasts. A strong and positive correlation ( $\mathrm{r}=0.921, \mathrm{p}=0.001)$ was found between the A. vera extract dosages and osteoblast count, whereas an inverse correlation was observed between the dosage and osteoclast count $(\mathrm{r}=-0.631, \mathrm{p}=0.001)$.

\section{DISCUSSION}

The study results revealed significant differences in osteoblast and osteoclast numbers between rats exposed to LPS alone and those exposed to LPS followed by $A$. vera ethanol extract. LPS stimulates osteoclast production, thereby resulting in tissue resorption. LPS functions as an endotoxin by binding to CD14 receptors in macrophages and monocytes. This binding induces the production of arachidonic acid (AA), which stimulates the secretion of cytokines such as IL- $1 \alpha$, IL-1 $\beta$, IL-6, TNF- $\alpha$, and PGE2 [11]. Prostaglandins and pro-inflammatory cytokines, which play important roles in bone pathology, are associated with bone destruction caused by localized chronic inflammation by increasing osteoclast formation, differentiation, and activation directly as well as by inhibiting osteoblast function [12]. Previous studies conducted by Indahyani et al. also proved that exposure to $E$. coli LPS leads to periodontitis.

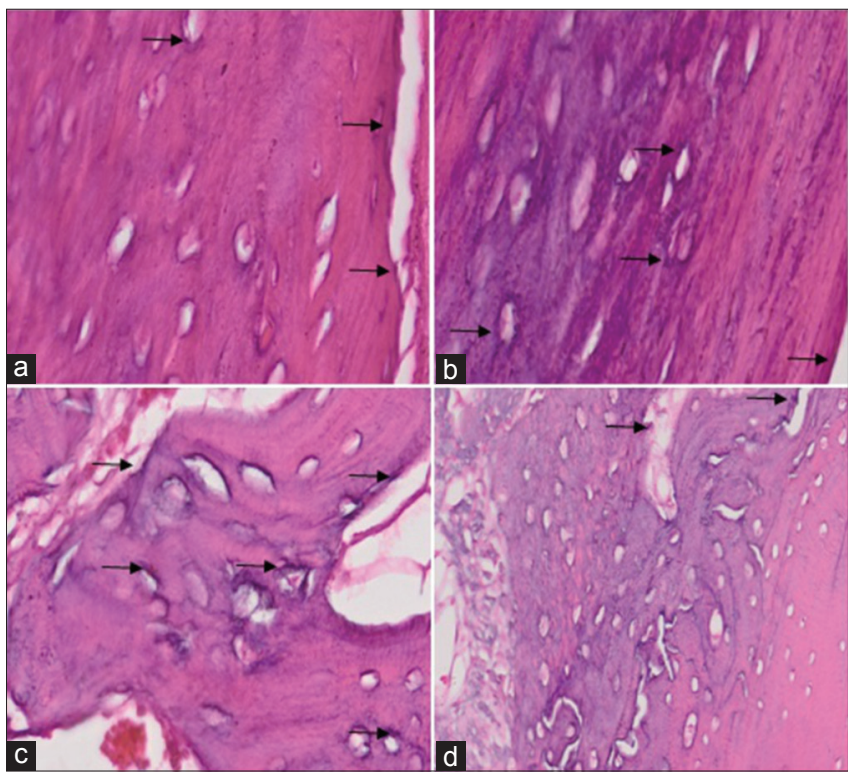

Fig. 3: (a-d) Histological comparison of osteoblast counts

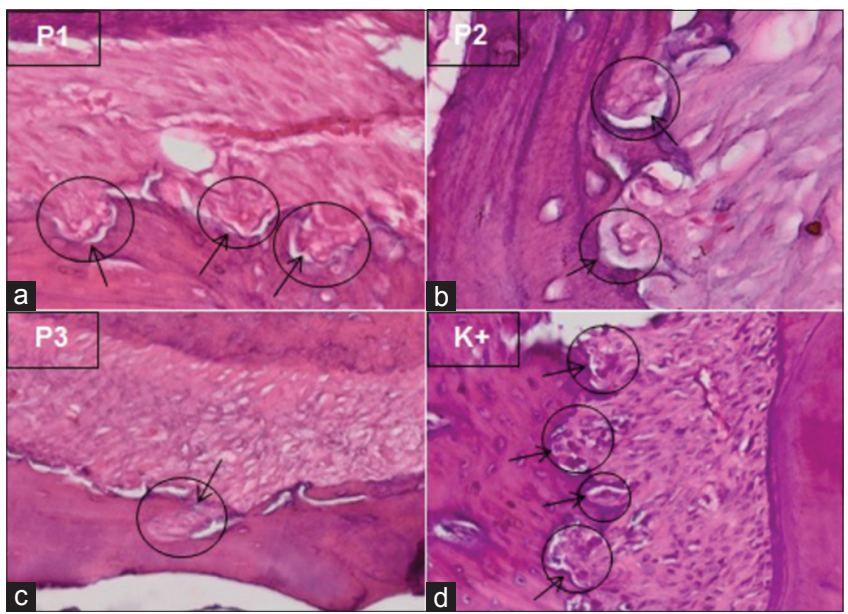

Fig. 4: Histological comparison of osteoclast counts. Description: (a) P1: Lipopolysaccharide (LPS) induction $+40 \mathrm{mg}$ of $A$. vera extract, (b) P2: LPS induction $+80 \mathrm{mg}$ of $A$. vera extract,

(c) P3: LPS induction $+160 \mathrm{mg}$ of $A$. vera extract, (d) K+: LPS induction alone 
The finding that the osteoblast numbers increase with increasing A. vera extract dosage corroborates with the hypothesis that acemannan can stimulate BMP2 expression and type 1 collagen fiber formation in periodontal tissue, thus facilitating bone regeneration by increasing osteoblast counts and decreasing osteoclast numbers in the alveolar bone. Acemannan is the largest polysaccharide capable of reducing inflammation through prostaglandin synthesis [13]. A. vera extract also inhibits AA-induced prostaglandin and thromboxane production through antagonism by eicosapentaenoic acid (EPA) [14,15]. In the body, EPA mostly interacts with AA metabolites. EPA is a polyunsaturated fatty acid that acts as a precursor for prostaglandin-3 (which inhibits platelet aggregation), thromboxane-3, and leukotriene-5. This substitution may result in the decreased release of pro-inflammatory signals, thus leading to decreased cytokine production.

A decrease in inflammatory mediator levels may also decrease osteoclastogenesis. Osteoblasts and stromal cells produce osteoprotegerin (OPG), which competes with RANKL for binding to RANK, thus suppressing osteoclast formation. When the OPG concentration exceeds that of RANKL, OPG binds to RANK and inhibits binding by RANKL. A study conducted by Kresnohadi demonstrated that acemannan can increase BMP2 expression and thereby lead to increased osteoblast formation and decreased expression of IL-1 $\beta$ and RANKL, which are markets of osteoclastogenesis and alveolar bone resorption [16].

The finding of the correlations between the A. vera extract dosage and osteoblast and osteoclast counts corroborates with data reported by Manoglas, as cited by Lindawati that BMP-2 stimulates osteoblastogenesis with increasing $A$. vera dosage [17]. In addition, A. vera also contains Vitamin $\mathrm{A}$, which has significant roles in cell differentiation and strengthening of collagen and Vitamin $\mathrm{C}$ bound stimulating type- 1 collagen, accumulation of osteoblasts, and matrix mineralization in osteoblasts. Vitamin $\mathrm{C}$ also maintains bone mass by stimulating osteoblast formation to promote the development of new bone and suppressing bone resorption by inhibiting osteoblast formation [18-20].

Linear regression analysis demonstrated that the $\mathrm{R}^{2}$ was 0.398 , thus illustrating that most effects of $A$. vera extract on osteoclasts were attributable to factors not analyzed in this study. These include the animal's conditions and the extract storage time, and other chemicals present in A. vera.

The hypothesis that $A$. vera extract can stimulate alveolar bone regeneration was successfully proved, as indicated by the increased number of osteoblasts and decreased a number of osteoclasts in LPS-treated rats.

\section{CONCLUSION}

A. vera ethanol extract appeared to promote alveolar bone regeneration in LPS-treated male rats based on the findings of increased osteoblastogenesis and decreased osteoclastogenesis. The A. vera extract dosage positively correlated with osteoblast counts and negatively correlated with osteoclast counts.

\section{REFERENCES}

1. WHO. The World Oral Health Report. WHO/NMH/ORH/03.2. Switzerland: World Health Organization; 2003.

2. Department of Health of Malang. Monthly Report of Community Health Center 2009. Malang: Department of Health; 2009.

3. Laine ML, Crielaard W, Loos BG. Genetic susceptibility to periodontitis. Periodontol 2000 2012;58:37-68

4. Carranza FA, Takei HH. Rationale for periodontal treatment. In: Newman MG, Takei HH, Klokkevold PR, editors. Carranza's Clinical Periodontology. $11^{\text {th }}$ ed. Missouri: Saunders Elsevier; 2010. p. 387-91.

5. Murray JA, Wilton JM. LPS from periodontal pathogen $P$. gingivalis prevents apoptosis of HL60-derived neutrophils in vitro. J Infect Immun 2003;71:7232-5.

6. IndahyaniDE, SantosoAL, Utoro T, Soesatyo MH.Lypopolisacharide(LPS) introduction during growth and development period of rat's tooth towart the occurance of enamel hypoplasia. Dent J 2007;40:85-8.

7. Baghban AA, Dehghani A, Ghanavati F. Comparing alveolar bone regeneration using bio-oss and autogenous bone graft in humas: A systemic review and meta-analysis. Iran Endod J 2009;4:125-30.

8. Wiedosari E. Role of natural immunomodulator (Aloe Chinensis in celullar and humoral immunity system). Wartazoa 2007;17:165-71.

9. Subramaniam MR, Gauri MU, Shivaraj BW. Bone morphogenetic proteins: Periodontal regeneration. J Med Sci 2013;5:161-8.

10. Furnawati I. Use of Aloe Vera. $7^{\text {th }}$ ed. Jakarta: Agro Medika Pustaka; 2006.

11. Stanshenko P. Interrelationship of Dental Pulp and Apical Periodontitis. Dental Pulpa. Inc. Chicago: Quintessence Publishing Co.; 2002.

12. Schwart Z, Goultschin J, Dean DD, Byan BD. Mechanism of alveolar bone destruction in periodontitis. The pathogenesis of periodontitis. Periodontology 2000 1997; 14:158-72.

13. Ramamoorthy L, Kemp M.C, Tizard IR. Acemmanan, a beta-(1,4)acetylated mannam, induces nitric oxide production in macrophage cell line RAW 264.7. Am Soc Farmacol Exp Ther 1996;50:878-4.

14. Saeed MA, Ahmad I, Yaqub U, Akbar S, Waheed A, Saleem M, et al. Aloe vera: A plant of vital significance. Q Sci Vis 2003;9:1-13.

15. Selvakumar R, Muralidharan NP. Comparison in benefits of herbal mouthwashes with chlorhexidine mouthwash: A review. Asian J Pharm Clin Res 2017;10:3-7.

16. Korver DR, Klasing KC. Dietary fish oil alters spesific and infalammatory immune responses in chicks. J Nutr 1997;127:2039-46.

17. Kresnoadi U. Toll-Like Receptor 2 as Signaling Pathway for Alveolar Bone Osteogenesis Induced with Combination of Aloe Vera and Graft. Dissertation. Surabaya: Airlangga University; 2012.

18. Pathak D. Vitamin C Protects, Maintains Healthy Bone Mass. Huston: Baylor College of Medicine; 2010.

19. Rastogi S, Iqbal MS, Ohri D. In vitro study of anti-inflammatory and antioxidant activity of some medicinal plants and their interrelationship. Asian J Pharma Clin Res 2018;11:195-202.

20. Joshua JM, Anikumar A, Cu V, Vasudevan DT, Surendran SA. Evaluation of the effect of different concentrations of Aloe vera on inflammation and re-epithelialization in diabetic ulcers in a rat model. Asian J Pharm Clin Res 2018;11:25-32. 